Research Paper

\title{
Three-dimensional Ultrasound Appearance of Pelvic Floor in Nulliparous Women and Pelvic Organ Prolapse Women
}

\author{
Tao Ying ${ }^{\bowtie}$, Qin Li, Lian Xu, Feifei Liu, and Bing $\mathrm{Hu}$ \\ Department of Ultrasound in Medicine, Shanghai Jiaotong University Affiliated Sixth People's Hospital, Shanghai 200233, People's Republic \\ of China.
}

$\triangle$ Corresponding author: Tao Ying, PhD, Department of Ultrasound in Medicine, Shanghai Jiaotong University Affiliated Sixth People's Hospital, Yishan Road 600 \#, Shanghai 200233, People's Republic of China. E-mail: yingtaomail@yeah.net, 1987714@sjtu.edu.cn. Phone: 0086-02164369181-8877. Fax: 0086-021-54488254.

(c) Ivyspring International Publisher. This is an open-access article distributed under the terms of the Creative Commons License (http://creativecommons.org/ licenses/by-nc-nd/3.0/). Reproduction is permitted for personal, noncommercial use, provided that the article is in whole, unmodified, and properly cited.

Received: 2012.07.06; Accepted: 2012.10.25; Published: 2012.11.09

\begin{abstract}
The present study investigated the morphology and structure of pelvic floor in 50 nulliparous and 50 pelvic organ prolapse (POP) women using translabial three-dimensional (3D) ultrasound. The levator hiatus in POP women was significantly different from that in nullipara women. In POP women, the size of pelvic floor increased, with a circular shape, and the axis of levator hiatus departed from the normal position in 36 (72\%) cases. The puborectalis was avulsed in $18(36 \%)$ cases and the pelvic organs arranged abnormally in $23(46 \%)$ cases. In summary, 3D ultrasound is an effective tool to detect the pelvic floor in POP women who presented with abnormalities in the morphology and structure of pelvic floor.
\end{abstract}

Key words: Three-dimensional ultrasound; Levator hiatus; Pelvic floor; Pelvic organ prolapse.

\section{Introduction}

Pelvic organ prolapse (POP) refers to a protrusion of one or more pelvic organs (bladder, rectum, uterus, vaginal vault, bowel) through vaginal fascia into the vagina. POP may cause different symptoms such as vaginal bulging (a major symptom), urinary or fecal incontinence, obstructed micturition or defaecation, sexual disorders and perineal pain [1-2]. POP affects many females with a mean prevalence of about 19.7\% (range: $3.4-56.4 \%$ ) in developing countries, and the prevalence of urinary incontinence with uterine prolapse, anterior vaginal wall prolapse and posterior vaginal wall prolapse was $31.3 \%, 59.7 \%$ and $44.8 \%$, respectively, in Beijing, a city of China.[3-4]. The etiology of POP is multifactorial. Delivery damage, chronic increasing abdominal pressure, congenital dysplasia of pelvic floor tissues are frequently found in clinical practice. It has been shown that age, parity and menopause are significantly associated with POP [5]. The pelvic floor supporting structures undertake the load of pelvic cavity and keep the pelvic organs above the levator hiatus in normal position. However, the damage of the pelvic floor supporting structures, especially the defects of levator ani muscle, may contribute to the occurrence of POP [6]. Of course, digital palpation is a good and easy method to detect avulsion of the levator ani muscle, while clinical evaluation should be performed together with imaging. Now the diagnosis of levator ani muscle trauma has become much easier with the help of modern imaging methods. Since the 1990s there has been description of levator ani trauma as visualized by magnetic resonance imaging (MRI). Three-dimensional modeling of MRI allows volumetric analysis to be performed and clarifies the spatial relationship of pelvic anatomic 
structures. Due to the superior spatial resolution and ability to identify different muscles in the pelvic floor, MRI has been a preferred method to assess the pelvic floor [7]. To date, the levator ani muscle trauma and the changes in the morphology of the pelvic floor after vaginal delivery and prolapse have been demonstrated using MRI [8-9]. But MRI has been of limited clinical use in the evaluation of pelvic floor disorders due to cost and access problems. Recently, with the technical improvement of three-dimensional (3D) ultrasound, 3D ultrasound has become a new tool in the detection of pelvic floor. Rendered 3D volume has the ability to visualize the axial plane with good spatial and superior temporal resolution, with volume datasets obtained per second [10]. Translabial 3D ultrasound can directly display the entire levator hiatus, puborectalis, previouslythe domain of MRI [11-14]. The agreement of features of the levator hiatus in 3D ultrasound and MRI was moderate as shown in a study of Kruger et al[13]. Dietz et al [15-16] proposed that, to detect the levator-urethra gap and asymmetric increase in the size of the levator hiatus with ultrasound can be used for the diagnosis of levator avulsion injury. The levator avulsion can be diagnosed reliably by tomographic ultrasound [17-18]. At the same time, ultrasound has the advantages of clinical convenience, easy access and safety throughout pregnancy. Moreover, patients with minimal discomfort can be examined at little expense and real time recording is also feasible. The present study aimed to detect the morphology and structure of the levator hiatus in POP women using the translabial 3D ultrasound and compare these features between POP and nulliparous women.

\section{Materials and Methods}

\section{Subjects}

This study was undertaken in Affiliated Sixth People's Hospital of Shanghai Jiaotong University from January 2011 to October 2011. This study was approval by the Ethics Committee of the hospital. Translabial 3D ultrasound was used to assess the pelvic floor in 50 POP women (POP group) and 50 nulliparous women (Nullipara group). All subjects gave their written informed consent before being enrolled into this study. The consecutive patients who would undergo prolapse repair were recruited into the POP group from the Department of Gynecology. The exclusion criteria for POP were $\mathrm{BMI} \geq 28$ and pelvic tumor with the diameter $\geq 3.0 \mathrm{~cm}$ (such as uterine myoma, ovarian tumor and fallopian tube tumor). Nulliparous women were also from the Department of Gynecology for the treatment of irregular menstrua- tion, vaginitis, pelvic inflammatory disease or infertility. Patients with a history of abortion, pelvic floor dysfunction and a family history, pelvic tumor (uterine myoma, ovarian tumor and fallopian tube tumor), pelvic trauma or surgery, chronic cough or constipation leading to increase in abdominal pressure and BMI $\geq 28$ were excluded from the Nulliparous group. All subjects were interviewed regarding the symptoms of urinary incontinence, prolapse or fecal incontinence with a standardized questionnaire [19]. Prolapse was examined according to the pelvic organ prolapse quantification (ICS POP-Q) staging system of International Continence Society ${ }^{[20]}$.

\section{Ultrasound examination}

The translabial ultrasound was performed in women in a supine position after voiding using a GE Kretz Volusion 730 system (GE Kretztechnik GmbH, Zipf, Austria) with RAB4-8P 3D ultrasound transducer. The transducer was covered with a glove. Imaging examination was performed in the midsagittal plane. Volume datasets were acquired at rest, on maximum Valsalva and maximum pelvic floor contraction for processing and subsequent analysis. Every patient was asked to push at her full stretch, each patient performed at least 3 Valsalva maneuvers, and the best one was used for evaluation. The 4D View software Version 1 (GE Medical systems, Kretztechnik $\mathrm{GmbH}$, Zipf, Austria) was used to acquire the images of entire levator hiatus in the axial plane. Then, the morphology and structure of the pelvic floor (the size and position of the levator hiatus, puborectalis and pelvic organs) were observed and compared between both groups.

The plane of minimum hiatal dimensions in the midsagittal view was identified by a line between the hyperechogenic posterior aspect of the symphysis pubis and the hyperechogenic anterior border of the puborectalis just posterior to the anorectal muscularis (represented by the single oblique line in Fig. 1) [12]. This line was then used to identify the plane of measurement in the axial plane. Figure 2 demonstrated the morphological findings on the minimal plane of the levator hiatus: levator hiatus area (HA), anteroposterior diameter of levator hiatus (AP) and left to right diameter of levator hiatus (LR). The levator hiatus, puborectalis and pelvic organs were observed in the 3D ultrasound. Two axile lines were defined to get a more accurate hiatal position. The symmetry axis of the bilateral pubic ramus was defined as the pelvic floor axis. The line linking the midpoint of the inner edge of the symphysis pubis and puborectalis was defined as the levator hiatus axis. If there is an intersection angle between the pel- 
vic floor axis and the levator hiatus axis, it is defined that the levator hiatus axis departed from the pelvic floor axis. Using a tomographic ultrasound imaging (TUI), a set of 8 parallel tomographic slices was obtained in the axial plane at intervals of $2.5 \mathrm{~mm}$ from $5.0 \mathrm{~mm}$ caudal to $12.5 \mathrm{~mm}$ cephalad of the plane minimum dimensions. The puborectalis avulsion was identified at the plane of minimal hiatal dimensions and two slices above it on the maximum pelvic floor contraction [21]. The puborectalis avulsion was diagnosed when there was loss of continuity between muscle and pelvic sidewall at least on one slice.
Avulsion includes full avulsion and partial avulsion. Full avulsionwas determined if the loss of continuity between muscle and pelvic sidewall is seen on all the 3 slices. Partial avulsion is diagnosed when loss of continuity between muscle and pelvic sidewall is seen on at least one slice. In normal condition, the urethra, vagina and rectum arranged tightly in a line in ventridorsal orientation inside the levator hiatus. If the other structures such as uterus, bladder, vagina vault, or intestinal canal other than the urethra, vagina and rectum were found in the hiatus, we defined the organs arranged abnormally.
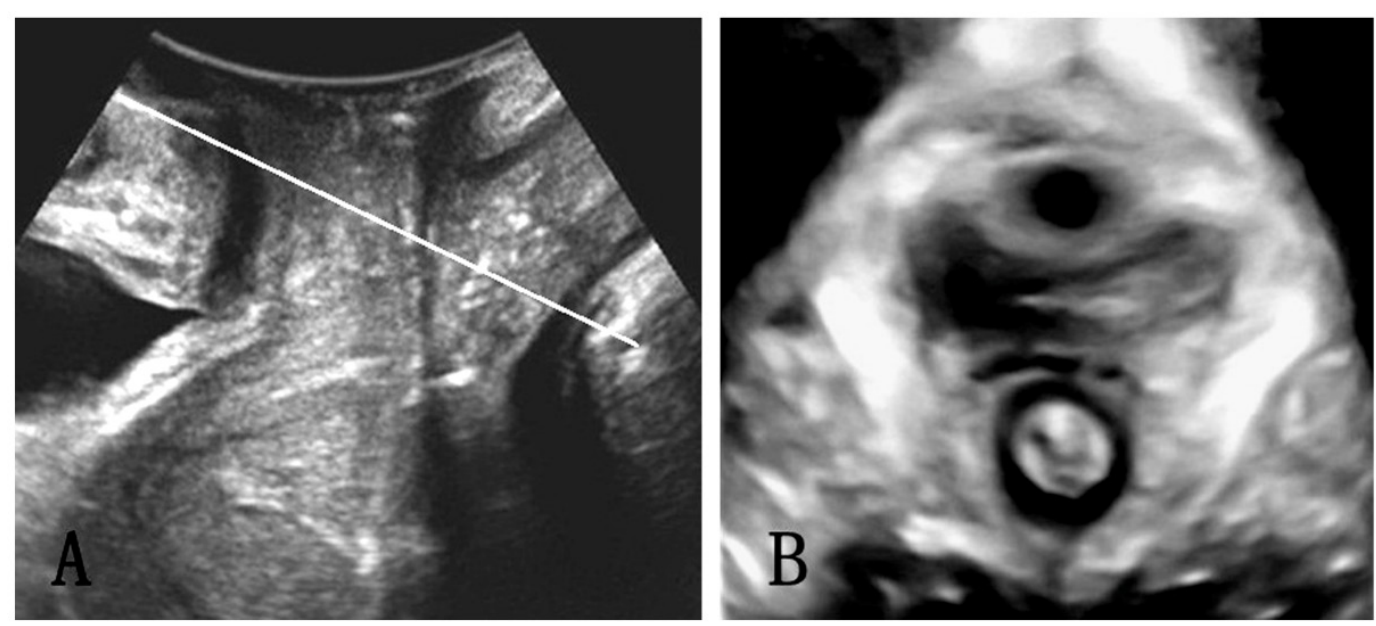

Figure I. The plane of minimal hiatal dimensions in the mid-sagittal plane (A) and axial plane (B).

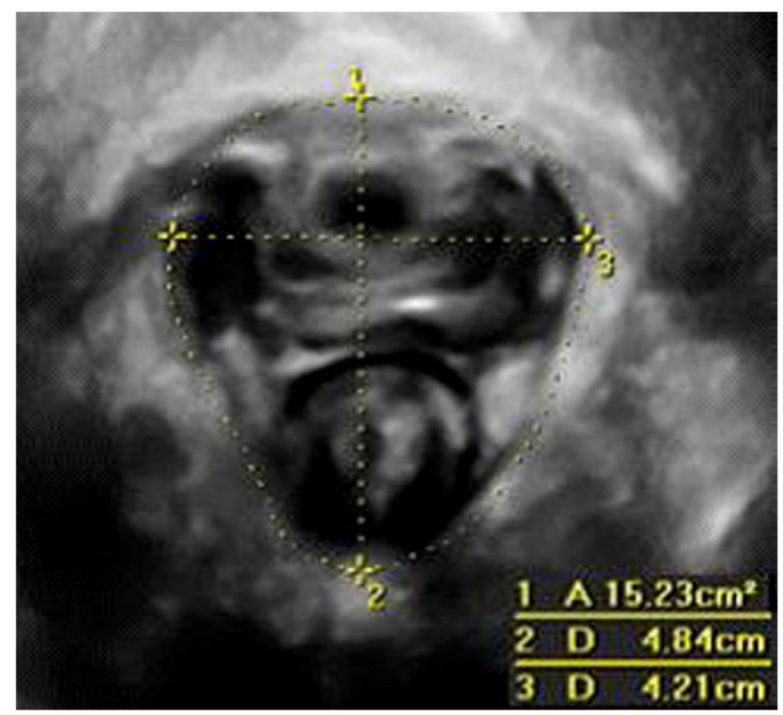

Figure 2. Measurement of levator hiatus in the minimal hiatal demensions: I, hiatal area (HA); 2, anteroposterior diameter (AP); 3, left-right diameter (LR). 


\section{Statistical analysis}

Statistical analysis was done with statistical analysis system 8.0 statistical program (SAS Institute Inc., Cary, NC, USA). The measured parameters were presented as mean positive and negative standard deviation (mean $\pm \mathrm{SD}$ ). The AP, LR and HA between both groups were compared using independent sample t-test. A value of $P<0.05$ was considered statistically significant.

\section{Results}

\section{General data}

The general demographics of patients in both groups are shown in Table 1. Complaints at presentation of POP women were stress incontinence $(15 \%)$, urge incontinence $(10 \%)$, symptoms of voiding dysfunction (such as hesitancy, a poor stream and straining to void; $45 \%$ ) and prolapse symptoms (90\%). The evaluation with clinical POP-Q in the POP group revealed Grade II POP in 12 women, Grade III POP in 20 women, and Grade IV POP in 18 women. On clinical assessment, 19 women suffered from anterior compartment prolapse, 23 suffered from central compartment prolapse and 8 suffered from posterior compartment prolapse. In the Nullipara group, patients were assessed as Grade 0 POP. Satisfactory volume datasets were obtained in all cases. Table 2 shows the measured parameters of two groups. Compared with the Nullipara group, the HA, AP and LR of the levator hiatus in the POP group were significantly increased at rest, on maximum Valsalva and maximum pelvic floor contraction $(\mathrm{P}<0.001)$.

\section{D ultrasound performance of pelvic floor in the Nullipara group}

Levator hiatus had compact structure outlined by the puborectalis and pubis. The pelvic floor axis and the levator hiatus axis overlapped (Fig. 3A). Puborectalis was symmetrical and continuous, and formed a "V"-shaped sling running from the pelvic sidewall towards the anorectal junction. On the ventral side, puborectalis closely attached to the interior edge of the pubic ramus without abnormal echo, and surrounded the posterior rectum on the dorsal side. At rest and on maximum Valsalva, the urethra, vagina and rectum arranged tightly in a line in ventridorsal orientation inside the levator hiatus, and the boundaries were clear.

\section{D ultrasound performance of pelvic floor in the POP group}

The levator hiatus increased in size and had an oval or round shape. In 36 (72\%) patients, the levator hiatus axis departed from the pelvic floor axis (Fig. 3B). The puborectalis in $18(36 \%)$ cases developed avulsion (Fig. 4), 10 had the unilateral right-sided avulsion (4 cases full avulsion, 6 partial avulsion), 6 had unilateral left-sided avulsion ( 2 cases full avulsion, 4 partial avulsion) and 2 had bilateral full avulsion. In the levator hiatus at rest, $23(46 \%)$ cases display abnormal organ arrangement. While on maximum Valsalva, 48(96\%) cases displayed abnormal organ arrangement, including 18 cases cystocele, 8 uterine prolapse, 15 vaginal vault prolapse and 7 rectocele.

Table I. General demographics of patients the POP group and the Nullipara group

\begin{tabular}{lll}
\hline Variables & POP women & Nullipara women \\
\hline Age $(\mathrm{y})$ & $64(40-83)$ & $23(18-35)$ \\
BMI $\left(\mathrm{kg} / \mathrm{m}^{2}\right)$ & $23.23 \pm 1.46$ & $21.92 \pm 2.23$ \\
Gravidity $(\mathrm{n})$ & $3(1-6)$ & 0 \\
Parity $(\mathrm{n})$ & $2(1-4)$ & 0 \\
\hline
\end{tabular}

Table 2. Measured parameters in the POP group and the Nullipara group (mean $\pm S D$ ).

\begin{tabular}{|c|c|c|c|c|c|c|c|c|c|}
\hline \multirow[t]{2}{*}{ Group } & \multicolumn{3}{|c|}{$\mathrm{AP}(\mathrm{cm})$} & \multicolumn{3}{|c|}{$\mathrm{LR}(\mathrm{cm})$} & \multicolumn{3}{|c|}{$\mathrm{HA}\left(\mathrm{cm}^{2}\right)$} \\
\hline & Rest & Valsalva & Contraction & Rest & Valsalva & Contraction & Rest & Valsalva & Contraction \\
\hline Nullipara & $4.31 \pm 0.72$ & $4.57 \pm 0.74$ & $4.08 \pm 0.74$ & $3.67 \pm 0.58$ & $3.98 \pm 0.61$ & $3.44 \pm 0.52$ & $11.22 \pm 2.61$ & $14.50 \pm 4.47$ & $9.18 \pm 2.65$ \\
\hline POP & $5.32 \pm 0.52$ & $5.92 \pm 0.54$ & $4.96 \pm 0.53$ & $4.43 \pm 0.51$ & $4.89 \pm 0.50$ & $4.07 \pm 0.52$ & $17.01 \pm 2.66$ & $22.76 \pm 3.72$ & $15.07 \pm 2.72$ \\
\hline$P$ values & $<0.001$ & $<0.001$ & $<0.001$ & $<0.001$ & $<0.001$ & $<0.001$ & $<0.001$ & $<0.001$ & $<0.001$ \\
\hline
\end{tabular}

Note: AP: anteroposterior diameter; LR: left to right diameter; HA: hiatus area. 

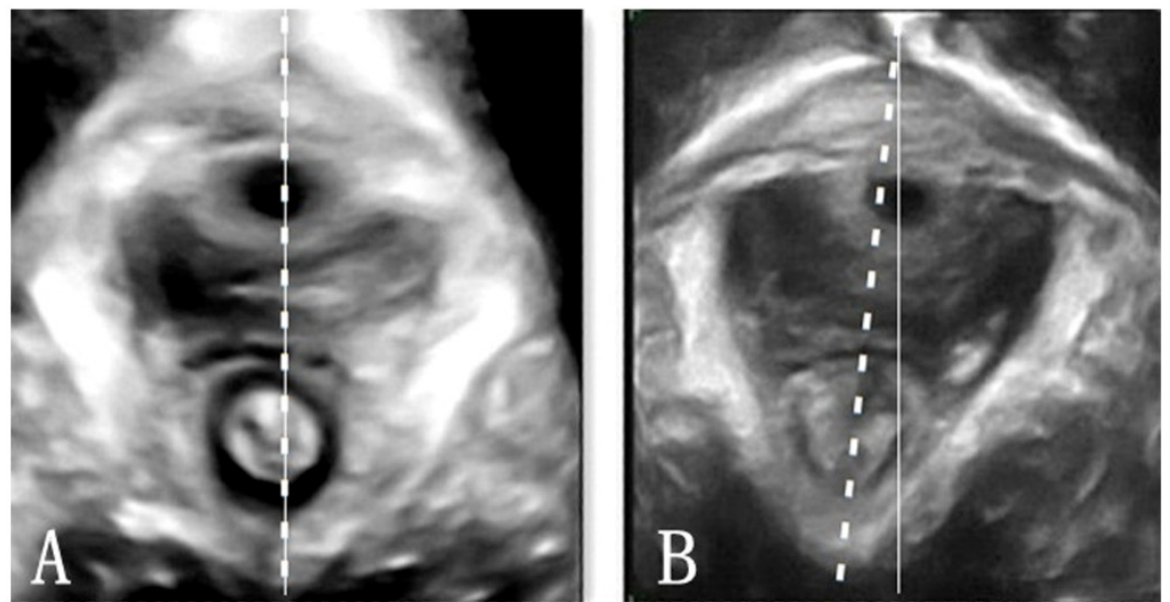

Figure 3. Position of the pelvic hiatus. A, the axis of levator hiatus and pelvic floor overlapped in nullipara patients; $B$, levator hiatus axis departed from the pelvic floor axis in POP woman. Dot line, levator hiatus axis; Solid line, pelvic floor axis.
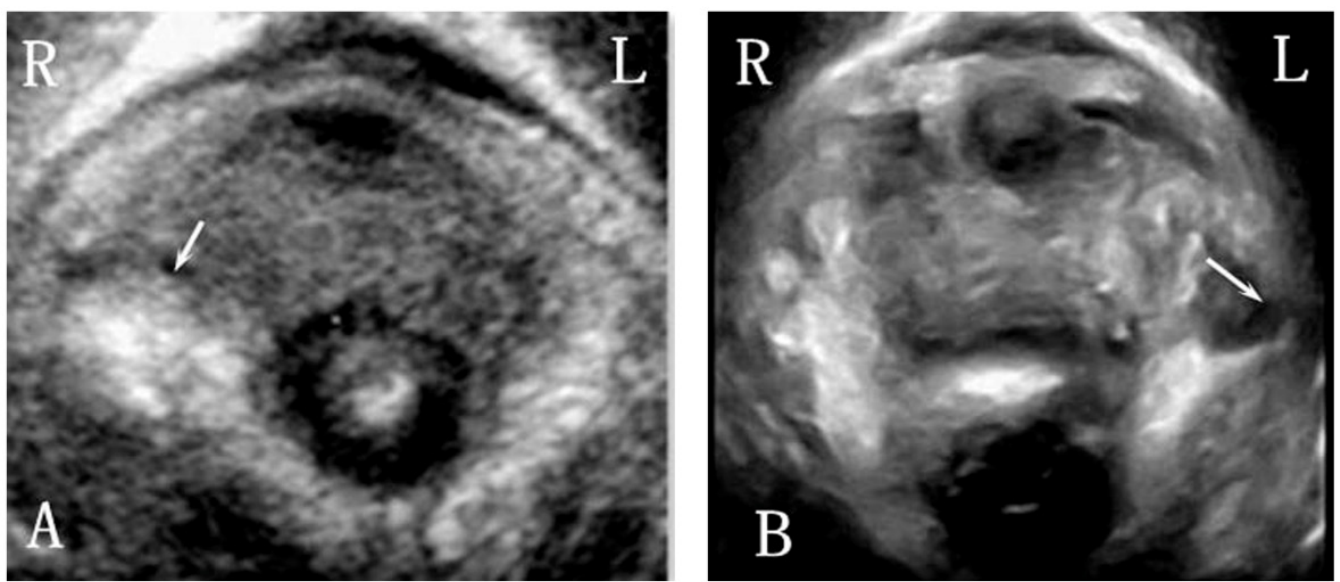

Figure 4. Right-sided and left-sided puborectalis avulsed in POP women.

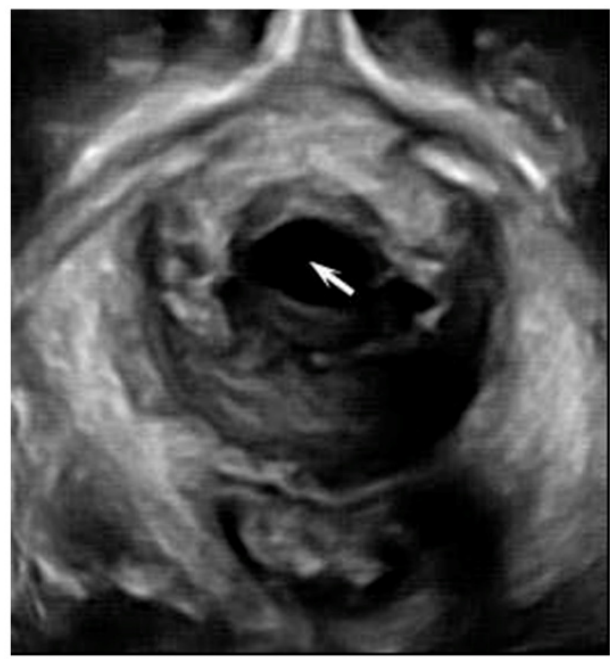

Figure 5. Cystocele (arrow) on 3D ultrasound imaging in a woman with POP. 


\section{Discussion}

In the present study, the morphology and structure of the levator hiatus were compared between the POP women and nullipara women. In the POP group, the size was larger, and the morphology tended to be circular, and the levator hiatus axis departed from the normal position. The puborectalis was avulsed, and the pelvic organs arranged abnormally.

The morphological changes and enlargement of the levator hiatus are the important characteristics of POP. It has been established that the size of the levator hiatus is related to the severity of prolapse [22-23], and may be used to predict the POP. In this study, results showed that the size of the levator hiatus of POP women was larger than that in nulliparous women at rest, on maximum Valsalva and maximum pelvic floor contraction. Vakili et al found that a widened genital hiatus correlated with an increase in surgical failures in the early postoperative period [24]. Thus, it may be important to assess the size of levator hiatus preoperatively and postoperatively.

Direct disruption of the pelvic floor muscles or damage to the pudendal nerves and its branches causing denervation of the muscles may lead to a compromised muscle function [25], which was in accordance with the morphological alternations in the puborectalis detected by 3D ultrasound. Dietz et al revealed that women with levator avulsion defects were more likely to develop pelvic prolapse [26]. In this study, the puborectalis avulsion was detected in 18 $(36 \%)$ cases, which was unilateral or bilateral in the POP patients. However, in the Nullipara group, abnormality in morphology was not found in puborectalis, which was consistent with previous studies [7,27]. These findings indicate that the abnormal morphology of the puborectalis is closely related to the development of POP.

In the Nullipara group, we found the pelvic floor axis and the levator hiatus axis overlapped, but in some POP patients the levator hiatus axis departed from the pelvic floor axis. The damage degree of puborectalis muscle is different in various patients. Some serious damage may lead to puborectalis avulsion which could be displayed by MRI or ultrasound. But some slight damage such as muscular flaccidity, diminished muscle contraction strength or inharmonious bilateral muscle contraction function may only be displayed the levator hiatus axis departed from the pelvic floor axis.

In the Nullipara group, the urethra, vagina and rectum arranged tightly in a line in ventridorsal orientation inside the levator hiatus, while in the POP group, the organs in the levator hiatus in maximum valsalva arranged abnormally in $48(96 \%)$ cases. The remaining 2 cases displayed normal arrangement may be due to the inadequate effort on pushing. Through comparing the images of the levator hiatus at rest and on maximum Valsalva, we could find more cases of abnormally arranged organs, thus performing adequate Valsalva maneuver is necessary to avoid false-negative result. At the same time, when checking the patients with higher grades of prolapse, it is essential not to exert undue pressure on the perineum so as to allow full development of pelvic organ descent.

In China, $B M I \geq 28$ was the criterion of obesity. Considering obesity may affect the penetration and resolution of ultrasound, we set $\mathrm{BMI} \geq 28$ as one of the exclusion criterion of both groups when recruiting the subjects. At the same time, the pelvic tumor with the diameter $\geq 3.0 \mathrm{~cm}$ was excluded due to the large pelvic tumor could affect the movement of pelvic organs. In addition, to get the healthy and standard nulliparous pelvic floor as the control, the criterion is very strict, the nullipara who had a history of abortion, pelvic floor dysfunction and a positive family history and so on were all excluded.

There are still some limitations in the present study. The age between both groups was significantly different. The pelvic floor may change with the increase in age and thus age is a confounding factor. More studies are needed in which the age as a confounding factor should be adjusted for further analysis. In addition, only ultrasound examination is done in the present study, and future study is required to compare the consistence among digital palpation, ultrasound examination and MRI in detecting the abnormalities of pelvic floor.

\section{Conclusion}

Under the translabial 3D ultrasound, the features of pelvic floor in POP women are significantly different from those in nullipara women, including the increased size, change in morphology, departed levator hiatus axis, avulsed puborectalis and abnormally arranged pelvic organs.

\section{Competing Interests}

The authors have declared that no competing interest exists.

\section{References}

1. Handa VL, Cundiff G, Chang HH, Helzlouer KJ. Female sexual function and pelvic floor disorders. Obstet Gynecol 2008;111:1045-52.

2. Slieker-ten Hove MC, Pool-Goudzwaard AL, Eijkemans MJ, et al. The prevalence of pelvic floor organ prolapse symptoms and signs and their 
relation with bladder and bowel disoders in a general female population. Int Urogynecol J Pelvic Floor Dysfunct 2009;20:1037-45.

3. Walker GJ, Gunasekera P. Pelvic organ prolapse and incontinence in developing countries: Review of prevalence and risk factors. Int Urogynecol J 2011;22:127-35.

4. Wang JL, Cao D, Zhang XH, et al. The prevalence of urinary incontinence and pelvic organ prolapse and its effects on the life quality of women in Beijing suburb. Chin J Clin Obstet Gynecol 2007;8:5-9.

5. Kim CM, Jeon MJ, Chung DJ, et al. Risk factors for pelvic organ prolapse. Int J Gynaecol Obstet 2007;98:248-51.

6. DeLancey JO, Morgan DM, Fenner DE, et al. Comparison of levator ani defects and function in women with and without pelvic organ prolapse. Obstet Gynecol 2007; 109: 295-302.

7. Margulies RU, Hsu Y, Kearney R, et al. Appearance of the levator ani muscle subdivisions in magnetic resonance images. Obstet Gynecol 2006;107:1064-9.

8. DeLancey JO, Kearney R, Chou Q, et al. The apperance of levator ani muscle abnormalities in magnetic resonance images after vaginal delivery. Obstet Gynecol 2003; 101:46-53.

9. Singh K, Jakab M, Reid WM, et al. Three-dimensional magnetic resonance imaging assessment of levator ani morphologic features in different grades of prolapse. Am J Obstet Gynecol 2003;188:910-5.

10. Dietz HP. Pelvic floor ultrasound. Curr Med Imaging Rev 2006;2:271-90.

11. Dietz HP. Ultrasound imaging of pelvic floor. Part II: three-dimensional or volume imaging. Ultrasound Obstet Gynecol 2004;23:615-25.

12. Dietz HP, Shek C, Clarke B. Biometry of the pubovisceral muscle and levator hiatus by three-dimensional pelvic floor ultrasound. Ultrasound Obstet Gynecol 2005;25:580-5.

13. Kruger JA, Heap SW, Murphy BA, et al. Pelvic floor function in nulliparous women using three-dimensional ultrasound and magnetic resonance imaging. Obstet Gynecol 2008;111:631-8.

14. Ying $\mathrm{T}, \mathrm{Hu} \mathrm{B}, \mathrm{Li}$ Q. Assessment of levator hiatus by $3 \mathrm{D}$ ultrasound volume contrast imaging in normal nulliparous. Shanghai Jiaotong Univ 2009;14:371-5.

15. Dietz HP, Abbu A, Shek KL. The levator-urethra gap measurement: a more objective means of determining levator avulsion? Ultrasound $\mathrm{Ob}-$ stet Gynecol 2008;32:941-5.

16. Dietz HP, Bhalla R, Chantarasorn V, Shek KL. Avulsion of the puborectalis muscle is associated with asymmetry of the levator hiatus. Ultrasound Obstet Gynecol 2011; 37:723-6.

17. Dietz HP. Quantification of major morphological abnormalities of the levator ani. Ultrasound Obstet Gynecol 2007;29:329-34.

18. Zhuang RR, Song YF, Chen ZQ, et al. Levator avulsion using a tomographic ultrasound and magnetic resonance-based model. Am J Obstet Gynecol 2011;205:232.e1-8.

19. Barber MD, Walters MD, Bump RC. Short forms of two condition-specific quality-of life questionnaires for women with pelvic floor disorders(PFDI-20 and PFIQ-7). Am J Obstet Gynecol 2005;193:103-13.

20. Bump RC, Mattiasson A, Bø K, et al. The standardization of terminology of female pelvic organ prolapse and pelvic floor dysfunction. Am J Obstet Gynecol 1996; 175:10-7.

21. Dietz HP, Bernardo MJ, Kirby A, et al. Minima criteria for the diagnosis of avulsion of the puborectalis muscle by tomographic ultrasound. Int Urogynecol J 2011;22:699-704

22. DeLancey JO, Hurd WW. Size of the urogenital hiatus in the levator ani muscles in normal women and women with pelvic organ prolapse. Obstet Gynecol 1998;91:364-8.

23. Ansquer Y, Fernandez P, Chapron C, et al. Static and dynamic MRI features of the levator ani and correlation with severity of genital prolapse. Acta Obstet Gynecol Scand 2006; 85:1468-75.

24. Vakili B, Zheng YT, Loesch H, et al. Levator contraction strength and genital hiatus as risk factors for recurrent pelvic organ prolapse. Am J Obstet Gynecol 2005;192:1592-8.

25. Sultan AH, Kamm MA, Hudson CN. Pudendal nerve damage during labor: prospective study before and after childbirth. Br J Obstet Gynaecol 1994;101:22-8.

26. Dietz HP, Simpson JM. Levator trauma is associated with pelvic organ prolapse. BJOG 2008;115:979-84.

27. Hoyte L, Damaser MS. Magnetic resonance-based female pelvic anatomy as relevant for materal childbirth injury simulations. Ann N Y Acad Sci 2007;110:361-76.

28. Ashton-miller JA, DeLancey JO. Functional anatomy of the female pelvic floor. Ann N Y Aacd Sci 2007;1101:266-96.

29. Huddleston HT, Dunnihoo DR, Huddleston PM, et al. Magnetic resonance imaging of defects in DeLancey's vaginal support levels I,II,III. Am J Obstet Gynecol 1995; 172:1778-82.
30. Dietz HP, Steensma AB. Posterior compartment prolapse on two-dimensional and three-dimensional pelvic floor ultrasound: the distinction between true rectocele, perineal hypermobility and enterocele. Ultrasound Obstet Gynecol 2005;26: 73-7. 